\title{
Le « tire-bouc », un jeu centrasiatique bien peu touristique
}

Goat-pulling: a Central Asian game hardly attractive for tourists

Carole Ferret et Camille Noûs

\section{OpenEdition}

Journals

Édition électronique

URL : http://journals.openedition.org/tourisme/3273

DOI : $10.4000 /$ tourisme.3273

ISSN : 2492-7503

Éditeur

Éditions touristiques européennes

Référence électronique

Carole Ferret et Camille Noûs, "Le « tire-bouc », un jeu centrasiatique bien peu touristique », Mondes du Tourisme [En ligne], 18 | 2020, mis en ligne le 01 mars 2021, consulté le 15 mars 2021. URL : http:// journals.openedition.org/tourisme/3273 ; DOI : https://doi.org/10.4000/tourisme.3273

Ce document a été généré automatiquement le 15 mars 2021.

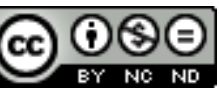

Mondes du tourisme est mis à disposition selon les termes de la licence Creative Commons Attribution - Pas d'Utilisation Commerciale - Pas de Modification 4.0 International. 


\title{
Le « tire-bouc », un jeu centrasiatique bien peu touristique
}

Goat-pulling: a Central Asian game hardly attractive for tourists

\author{
Carole Ferret et Camille Noûs
}

Puis, d'un seul coup, les lanières lestées de plomb se levèrent comme un peuple de reptiles sifflants au-dessus des bonnets de fourrure, un hurlement d'une sauvagerie démente, alliage de toutes les clameurs, déferla sur le plateau et la dépouille animale fut recouverte par la masse des hommes et des bêtes. Par une transformation si soudaine

que personne n'en avait pu saisir l'instant, la troupe ordonnée et solennelle n'était plus que tumulte, frénésie, prodigieux tourbillon. Huées, invectives, menaces inarticulées... Cravaches qui cinglaient, déchiraient naseaux et visages... Flux et reflux... Chevaux cabrés de toute leur hauteur sur l'enchevêtrement des corps et des poitrails... tchopendoz accrochés, suspendus au flanc de leur monture, le front dans la poussière, les ongles griffant, raclant le sol pierreux, afin de trouver le bouc décapité, et le saisir, et l'arracher.

(Kessel, 1967, p. 89-90)

1 Dans quelle mesure un tel jeu équestre est-il susceptible d'attirer des touristes? Les pays d'Asie centrale, devenus indépendants depuis la dislocation de l'URSS en 1991, peuvent-ils espérer tirer profit de l'exotisme de ce spectacle, représentation stylisée et caricaturale de leur vie pastorale, pour les aider à devenir une destination prisée par des touristes friands d'émotions et d'authenticité ? J'essaierai de répondre à ces interrogations en montrant les différentes formes du jeu de "tire-bouc» en Asie centrale, leur évolution liée à un processus de «sportification » et les obstacles qui 
s'opposent à sa mise en tourisme, à partir des matériaux de terrain que j'ai rassemblés sur ce sujet entre 1994 et 2017, principalement au Kazakhstan, mais aussi au Kirghizstan, en Ouzbékistan et dans l'Altaï russe.

\section{Le jeu de tire-bouc}

2 Le tire-bouc est une sorte de rugby à cheval, pratiqué par des peuples turciques d'Asie centrale ${ }^{1}$, où le cadavre d'un caprin tient lieu de ballon. Des cavaliers s'efforcent de s'en emparer, par terre ou en l'arrachant à un adversaire, et de l'emporter en se dégageant de la mêlée pour marquer un essai. L'ampleur et les règles du jeu varient grandement : individuel ou en équipe, avec ou sans but(s), rassemblant de quelques cavaliers à plusieurs centaines. C'est un jeu viril et violent, organisé à l'occasion de fêtes familiales ou publiques. Son nom varie selon les langues : kôkpar en kazakh, kôkbôrù ou ulak en kirghize, uloķ en ouzbek et en tadjik, buzkaši en tadjik, autrement dit le bouzkachi que l'on connaît en occident comme un jeu afghan, décrit par Kessel.

Fig. 1. Asie centrale

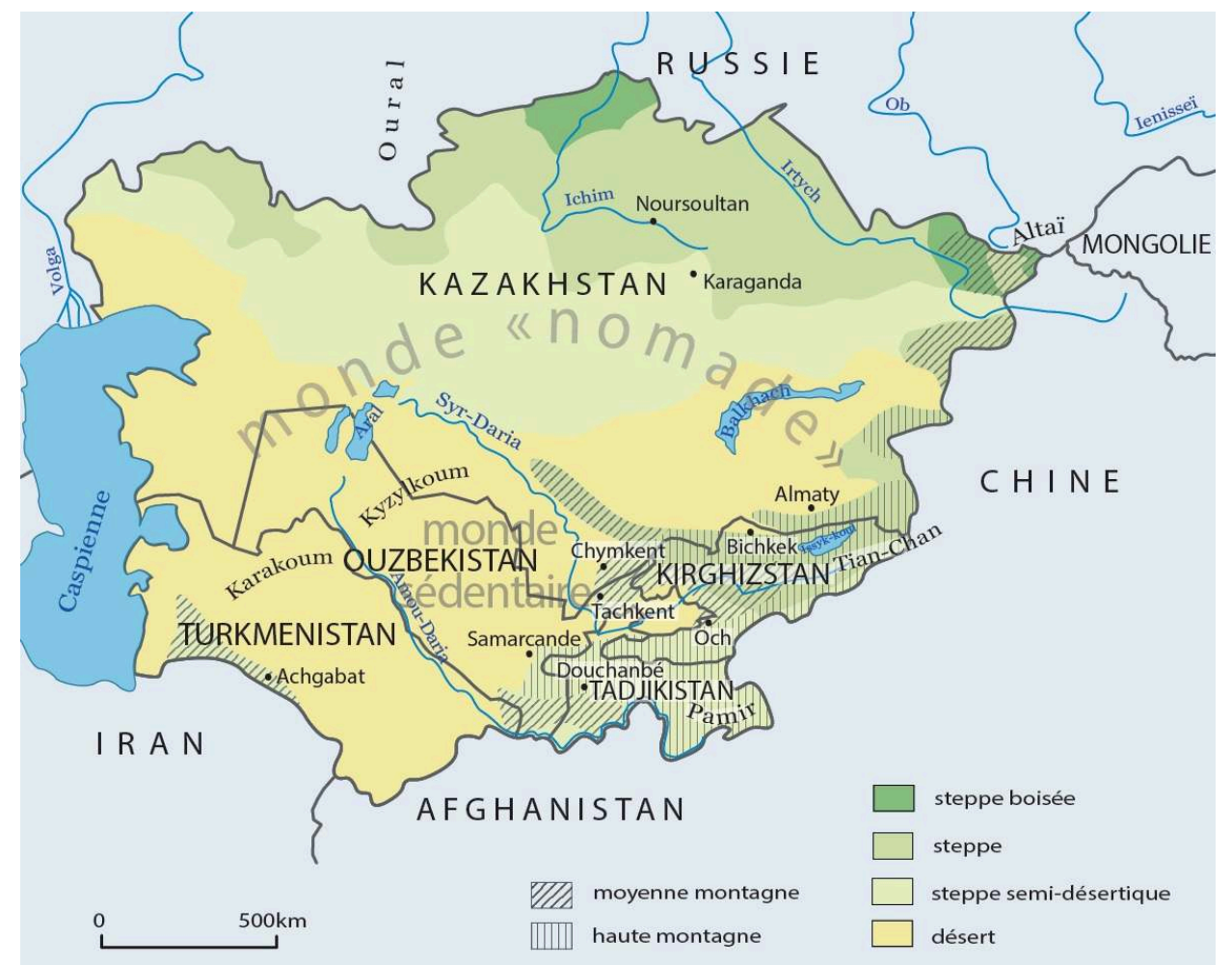

Carte C. Ferret

En Asie centrale soviétique, le tire-bouc (kozlodranie en russe) a été intégré dans l'ensemble des jeux sportifs équestres "nationaux et populaires». Il a d'abord été déconsidéré, et même un moment proscrit, dans les années 1960, au moins dans ses formes les plus visibles, car ce jeu dispendieux accompagnait autrefois de grandes fêtes accusées de servir le pouvoir féodal des plus riches. Mais, en fin de compte, le pouvoir soviétique n'a pas tant interdit le kôkpar qu'il a cherché à le transformer en une compétition d'un autre genre, en le poliçant et en l'intégrant dans un folklore soviétique (Ferret, 2018). Comme nombre d'autres activités ludiques dans le monde, le 
tire-bouc a donc suivi en URSS un mouvement global de sportification, qui a vu la transformation de jeux populaires, souvent chaotiques et violents, en des sports, obéissant à des règles codifiées (Elias et Dunning, 1994).

\section{L'invention d'une identité touristique}

4 Après la dislocation de l'URSS en 1991, les jeux équestres traditionnels ont connu une vogue sans précédent en Asie centrale. En effet, les cinq républiques devenues indépendantes sont des États jeunes, qui doivent inventer une histoire nationale, en valorisant un patrimoine qui leur serait propre. Or l'Asie centrale est classiquement divisée en deux ensembles antagonistes et complémentaires : le monde sédentaire des oasis et le monde nomade des steppes. De ce dernier se revendiquent ouvertement le Kazakhstan et le Kirghizstan qui, ensemble et concurremment, se sont proclamés héritiers des nomades des steppes eurasiatiques. Par l'usage du cheval comme monture et de la chèvre comme proie, le tire-bouc se place apparemment du côté nomade et il compte parmi les us représentatifs de cette aire culturelle.

Face à l'Ouzbékistan, riche de Boukhara, Samarcande et Khiva, le Kazakhstan et le Kirghizstan ne peuvent rivaliser par leur patrimoine architectural pour attirer des touristes, aussi mettent-ils en avant leur environnement naturel et leur culture nomade (Werner, 2003). C'est pourquoi le choix de l'écotourisme s'imposait, dans des paysages de steppes ou de montagnes, avec une orientation sportive, par la randonnée, ou culturelle, par la valorisation de la vie pastorale «traditionnelle » (Pabion-Mouriès, 2013 ; Hallé et Raspaud, 2012 ; Tiberghien et Xie, 2018).

6 L'enjeu touristique n'est pas le même pour ces deux pays, dont l'envergure et la richesse diffèrent. Le nombre de touristes y est comparable, bien que difficile à évaluer parmi les visiteurs étrangers (4,5 millions en 2014 au Kazakhstan; 1,4 million au Kirghizstan en 2017). Mais le tourisme n'est qu'une ressource secondaire au Kazakhstan, permettant de diversifier une économie dominée par la production d'hydrocarbures, où le PIB dépassait les 170 milliards de dollars en 2018 (9331 \$/ habitant), alors qu'il est crucial pour le Kirghizstan, qui compte trois fois moins d'habitants et dont les ressources sont bien plus modestes (PIB : 8 milliards de dollars en 2018 , soit $1300 \$ /$ habitant $)^{2}$.

7 Dans ces deux pays, la revendication du nomadisme pastoral comme fondement de l'identité nationale est empreinte d'ambiguïté, prise entre glorification récente et dénigrement ancien du nomadisme, lié à un paradigme évolutionniste le rendant incompatible avec la modernité (Ferret, 2016). La patrimonialisation du nomadisme se focalise sur quelques emblèmes, tels que la iourte de feutre ou le cheval, quitte à ériger des iourtes immobiles, en béton, en ignorant l'essence du nomadisme, à savoir la mobilité résidentielle des éleveurs. Qu'il soit dénigré comme naguère ou glorifié comme à présent, le nomadisme reste relégué dans un passé révolu.

8 La patrimonialisation du nomadisme s'est accompagnée d'une promotion des jeux équestres centrasiatiques dits traditionnels, tandis que les sports hippiques allogènes, catégorisés comme russes ou occidentaux, étaient délaissés (Ferret, 2008). Au premier rang de cette dizaine de jeux équestres kazakhs et kirghizes (Gunner et Rahimgulov, 1949 ; Fedotov, 1980 ; Simakov, 1984) se trouvent la course hippique de longue distance (bäjge en kazakh, čabyš en kirghize) et le tire-bouc. Ils ont chacun leurs spécialistes cavaliers, entraîneurs, connaisseurs, public d'habitués - et leur aire de diffusion propre, 
tandis que les autres jeux équestres ont une dimension plus anecdotique et folklorique. Depuis 1990, tire-bouc et bäjge ont connu un fort développement, désormais encouragés par les autorités gouvernementales en tant que manifestations de la culture nationale. Ils sont organisés par des administrations publiques lors de fêtes calendaires, nationales, ou des jubilés, et par des personnes privées dans le cadre de fêtes familiales. Parallèlement, le processus de sportification de ce jeu s'est amplifié dans la période postsoviétique, ce qui est aussi une façon de le sauvegarder tout en le dénaturant.

Si cette promotion des jeux équestres sert et accompagne un mouvement de construction d'une identité nationale, fondé sur la patrimonialisation du nomadisme pastoral, est-elle également susceptible d'attirer des touristes, en particulier de l'étranger proche et lointain?

\section{Un jeu exotique, mais pas vraiment nomade}

10 Par son exotisme, le tire-bouc offre un spectacle original et impressionnant, qui semble être un bon candidat à une exploitation touristique (Tiberghien et Xie, 2018, p. 9). Manifestant une violence âpre et rude où se mêlent confusément hommes et chevaux, il captive par son étrangeté. Chez les spectateurs, il suscite à la fois la fascination et l'incompréhension, deux sentiments qui semblent opposés mais s'entretiennent mutuellement.

11 En offrant une mise en scène caricaturale de l'activité pastorale, où s'opposent avec violence des cavaliers virils, dont l'allure correspond parfaitement au stéréotype du «nomade des steppes», le tire-bouc propose une image d'Épinal du monde centrasiatique. Sauvages et belliqueux, le šabandoz (cavalier de tire-bouc), le guerrier des armées de Gengis Khan et le moudjahidine afghan participent d'une même imagerie. Le tire-bouc apparaît comme le reflet de la mentalité et des rudes mœurs centrasiatiques (Azoy, 2002). Comme le rodéo pour le cow-boy, il est une mise en scène idéalisée qui héroïse le passé nomade (Lawrence, 1982, p. 6 ; Saumade, 2011) tout en ayant peu à voir avec les réalités quotidiennes du pastoralisme. Il est un des ingrédients du patrimoine national promus dans le cinéma de la propagande gouvernementale (Isaacs, 2015, p. 405) et dans les spots publicitaires (Werner, 2000, p. 125).

12 Cette représentation stylisée de l'identité nationale peut être à usage interne, destinée aux Kazakhstanais ou Kirghizstanais, et externe, pour les étrangers, qui apprécient la couleur locale et pour lesquels il représente une "exciting custom» (Werner, 2003, p. 145). Ainsi, les personnels des ambassades étrangères à Tachkent, en Ouzbékistan, qui ne sont pas exactement des touristes mais dont la sociabilité est néanmoins peu intégrée à la vie locale, se plaisent à aller voir un tire-bouc le week-end, non loin de la ville. P. Parlebas (2003b) cite le tire-bouc parmi les jeux les plus typiques au monde, montrant que « les peuples se distinguent autant par leurs jeux que par leurs langues ».

13 Cependant, faire du tire-bouc un « jeu nomade » relève pour une part d'un malentendu, voire d'une supercherie. En effet, cette qualification, si on la réserve comme il se doit à des situations de mobilité résidentielle liée au pastoralisme, ne résiste pas à un examen attentif des caractéristiques techniques de ce jeu, comparé à la course hippique de longue distance (cf. fig. 2). 
Fig. 2. Bäjge

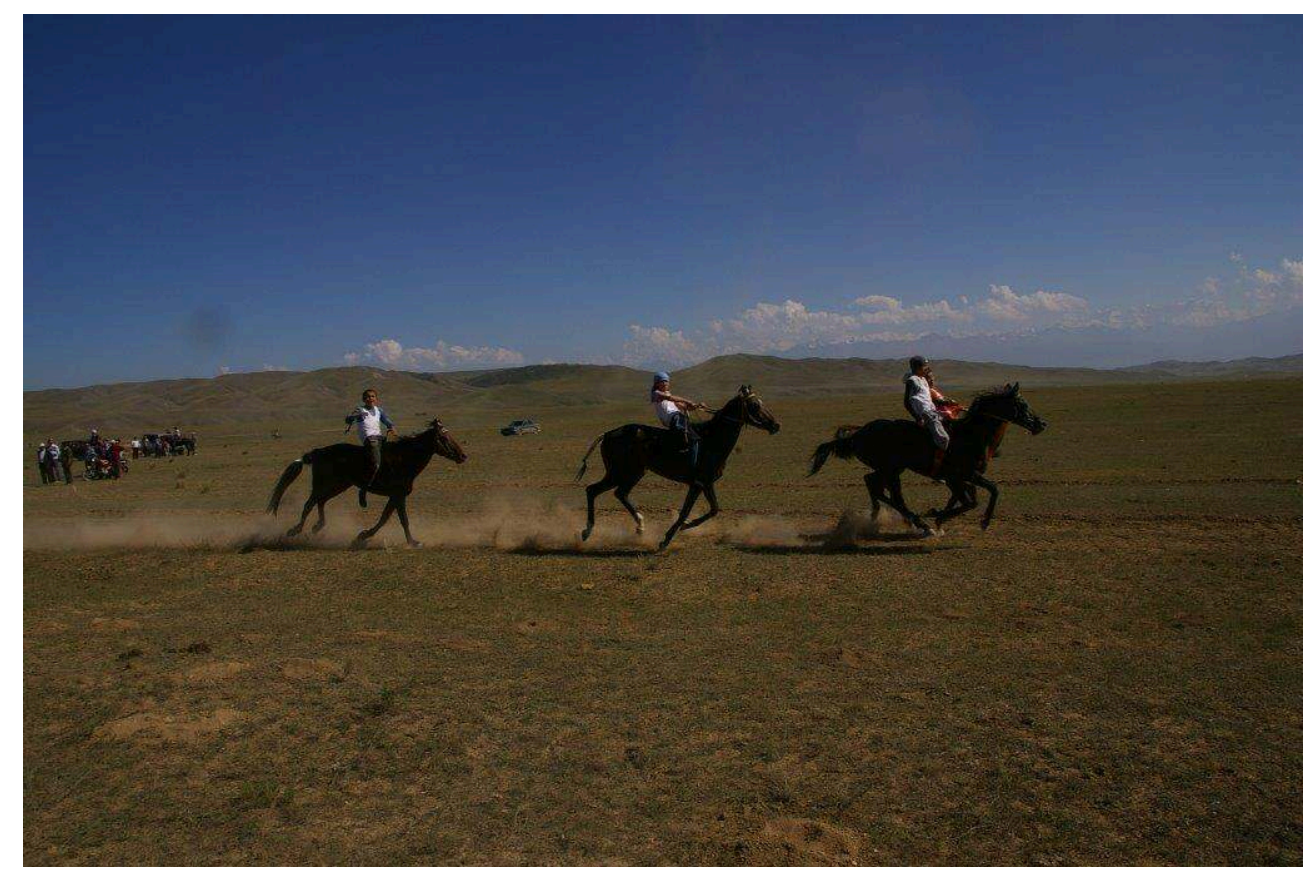

Photo C. Ferret, août 2013, sud-est du Kazakhstan hongres, courent dès un an et demi, par catégories d'âge mais sans séparation des sexes. Secs, voire maigres, les coursiers sont de format longiligne, monté par des garçons légers, souvent à cru. À l'inverse, les chevaux de tire-bouc sont de puissants et robustes étalons adultes, âgés de 5 à 15 ans, spécialement entraînés. Il n'y a pas de catégories d'âge mais une homogénéité sexuelle, car seuls des entiers sont censés être assez vaillants pour entrer dans la mêlée. Ils sont de modèle assez lourd et ont les aplombs solides, pour tenir le coup dans la mêlée et résister aux coups de boutoir de leurs congénères. Ils sont montés par des d'hommes d'âge mûr, costauds et solidement équipés : casque ou chapka pour protéger la tête des coups, habits molletonnés, lourdes bottes à talons. La rapidité importe dans les deux jeux, mais les qualités requises diffèrent: légèreté pour la course, force pour le tire-bouc. En témoigne l'autre dénomination des ŝabandoz, appelés éloquemment « lutteurs » en kazakh.

choix des étalons montre qu'il ne s'agit pas d'un jeu typiquement nomade, contrairement à l'image qui en est donnée, car les pasteurs nomades montent habituellement des hongres, laissant les juments et les étalons pour la reproduction dans les troupeaux en pacage libre. Ce sont des sédentaires qui montent des étalons, en particulier dans l'aire d'influence iranienne. Ils les gardent dans des écuries au moins une partie de l'année et les nourrissent avec des céréales, pratiquant un élevage plus intensif que les nomades.

L'aire de diffusion privilégiée du tire-bouc confirme cette impression: sud du Kazakhstan et du Kirghizstan, Ouzbékistan, Tadjikistan, nord de l'Afghanistan sont des régions de tradition sédentaire. Plus que le tire-bouc, c'est le bäjge, pratiqué dans le centre, le nord et l'est du Kazakhstan et du Kirghizstan, régions du nomadisme centrasiatique, qui mérite d'être qualifié de jeu nomade. On pourrait grossièrement diviser l'Asie centrale en deux: un monde d'influence nomade, essentiellement 
steppique, qui correspond à celui du bäjge, en forme de croissant couvrant la majeure partie du Kazakhstan et allant du nord du Turkménistan au centre du Kirghizstan; et un monde sédentaire, celui du tire-bouc, qui forme un noyau central et méridional, fait de déserts et d'oasis, situé au sud du Syr-Daria (cf. fig. 1). Des pasteurs mobiles qui élèvent les chevaux en troupeaux jouent parfois au tire-bouc, mais lors de petites parties improvisées sur les pâtures, où les cavaliers montent des hongres qui servent au gardiennage des troupeaux, sans entraînement ni enjeu particuliers, alors que les grands tire-bouc réunissant des dizaines ou des centaines de participants ont lieu chez les sédentaires. C'est ce type-ci de compétition, agrémentant certains toj (grandes fêtes centrasiatiques), qui correspond à la forme canonique du tire-bouc et que je décrirai ici.

L'exotisme du jeu se manifeste dans son déroulement mais aussi, plus discrètement, dans une méthode d'entraînement très originale que j'ai découverte en m'entretenant avec plusieurs éleveurs (Ferret, 2018). À des périodes d'engraissement, où le cheval est suralimenté tout en restant immobile, succèdent des périodes d'amaigrissement, où sa ration est réduite alors qu'il est soumis à un exercice physique croissant. En outre, son alimentation est parfois enrichie d'ingrédients peu ordinaires pour des herbivores : lait, œufs, thé, opium. Ce régime spécifique, qui repose sur une conception particulière de la physiologie, fondée sur l'opposition du chaud et du froid, du sec et de l'humide (Ferret, 2004), est contraire aux principes d'une alimentation équilibrée, proportionnée à l'effort, tels que nous les connaissons usuellement pour les entraînements sportifs.

\section{Un jeu brutal}

18 Le tire-bouc offre donc un spectacle exotique, mais sans doute un peu trop. S'il est douteux qu'une photo de moudjahidine puisse être présentée comme un argument de vente sur une brochure touristique, il apparaît aussi, dans une moindre mesure, que le spectacle du tire-bouc heurte les sensibilités des touristes par la violence déployée, tant entre les hommes qu'envers les animaux.

Qualifié de "sport le plus brutal au monde » (Barclay, 1980, p. 318), le tire-bouc a fait l'objet de descriptions plus apocalyptiques les unes que les autres.

Il faut observer cette foule bestiale de cavaliers, entendre les piétinements, les hululements, les ronflements et les lourdes exhalations de cette masse uniforme, mêlée d'animaux et d'hommes ayant perdu la tête, la pluie des terribles coups de fouet assénés à droite et à gauche, pour comprendre toute la sauvagerie de ce divertissement primitif et enragé. Les crânes brisés, les bras et les jambes cassés ne sont pas rares. Les cavaliers tués et les chevaux estropiés sont les compagnons inévitables et constants de ce sport du Turkestan [...] Dans leur course folle, les participants tentent de ravir les uns aux autres le trophée, qui passe de main en main, jusqu'à ce que leur piétinement fasse progressivement disparaître les entrailles du bouc tombées à terre, les morceaux de viande fraîche et de laine arrachée, et que l'un d'eux, plus chanceux, parvienne à s'extirper de la mêlée dense, tenant à la main une masse informe ensanglantée... (Kolosovskij, 1910, p. 111-112).

Cette violence s'exerce d'abord contre l'instrument du jeu, le kôkpar, qui est le cadavre d'un jeune caprin, d'un bouc castré, ou plus rarement d'un veau, dont on a coupé la tête et l'extrémité des pattes, parfois rempli de sable. Ce «ballon » avec lequel on joue est donc très lourd : 30 à $40 \mathrm{~kg}$ et en piteux état après une ou deux heures de jeu, à force d'être piétiné et écartelé. Si l'animal est maintenant abattu et décapité avant d'être mis en jeu, quelques témoignages attestent qu'autrefois, il pouvait être encore vivant en début de partie (Burnes, 1842, p. 202) et même d'autres, moins dignes de foi, qu'il aurait 
pu s'agir d'infortunés prisonniers de guerre transformés en "gelée d'hominidés " (Dupree, 1980, p. 218). Plusieurs auteurs interprètent son rôle comme celui d'une victime expiatoire, l'assimilant à la figure du bouc émissaire, réceptacle passif qui concentre sur lui toutes les violences. Malgré son évidence, assise sur l'espèce caprine du kôkpar, cette interprétation doit être examinée avec circonspection, tout comme l'analogie du tire-bouc avec un sacrifice, qui concorde mal avec le déroulement effectif du jeu sur le terrain et l'absence de dédicataire.

Cette brutalité s'exprime ensuite entre les participants, hommes et chevaux. La lutte pour s'emparer du kôkpar est violente et acharnée. Les coups pleuvent sur les bêtes et les hommes. Le šabandoz frappe son propre cheval pour lui faire oublier sa peur et le faire entrer dans la mêlée, ou pour le faire galoper plus vite ; il frappe les montures de ses adversaires, pour les écarter ou les faire démarrer en lui abandonnant leur proie ; il frappe aussi ses adversaires. L'arme du šabandoz est son fouet, composé d'un manche court en bois et d'une mèche en cuir de même longueur. À leur jonction, une pièce de cuir permet de tenir le fouet entre les dents, position emblématique qui confère au šabandoz un air de sauvagerie sans égal - même si paradoxalement, elle correspond aux rares moments où il ne frappe personne...

Fig. 3. Kôkpar tenu par un šabandoz, fouet entre les dents

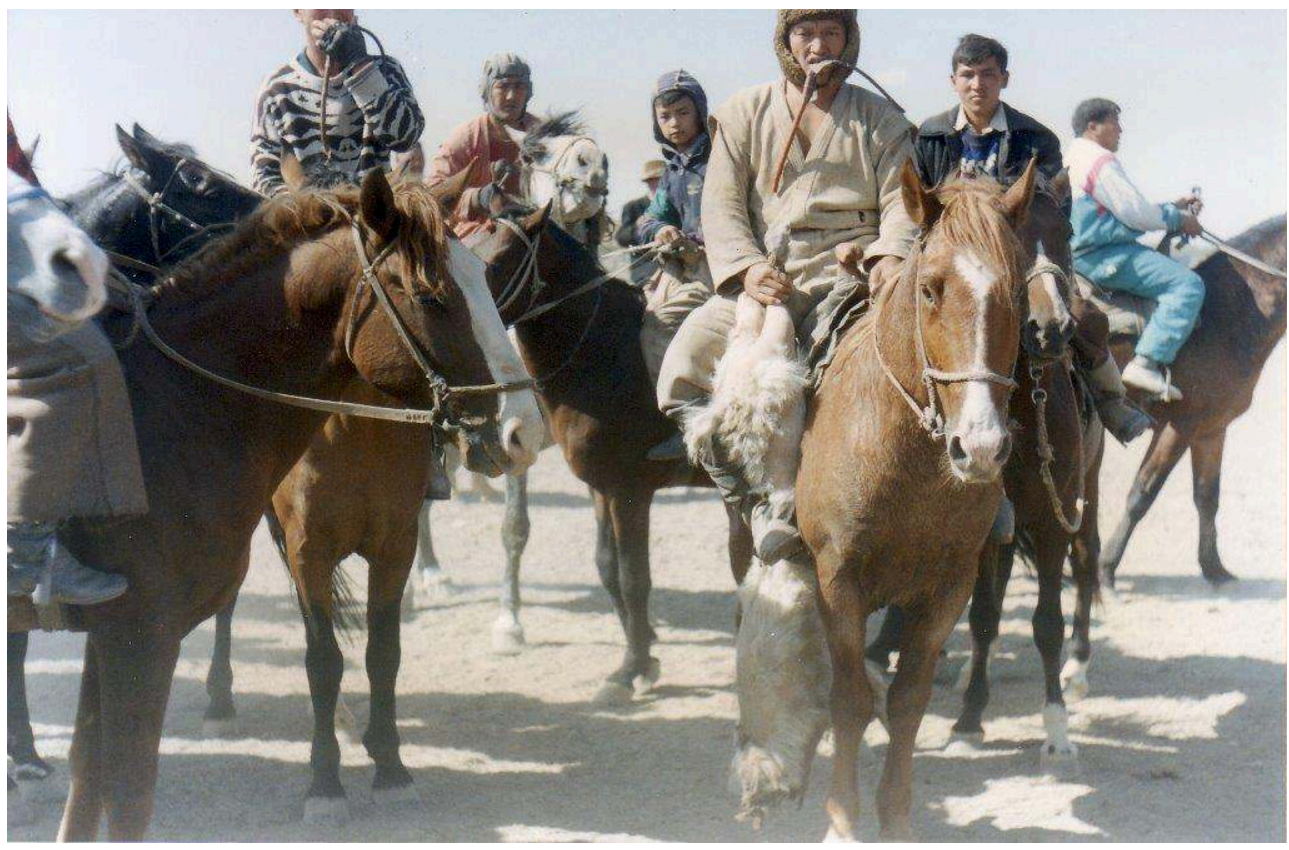

Photo C. Ferret, octobre 1994, sud du Kazakhstan

Les chutes sont fréquentes, mais les accidents mortels assez rares au regard de la violence déployée. En effet, les chevaux évitent, dans la mesure du possible, de piétiner un être vivant tombé à terre. Depuis 1994, j'ai vu moins de chevaux mourir sur les terrains de tire-bouc qu'à l'issue des bäjge, épuisés à l'arrivée. Mais les blessés et les contusionnés sont nombreux. Les vidéos, qui abondent désormais sur Internet, regorgent de scènes violentes et de chutes impressionnantes.

Le tire-bouc est donc dangereux pour les participants, humains et animaux, mais parfois aussi pour le public, car le terrain de jeu n'est pas délimité. Les spectateurs, à pied ou à cheval, doivent souvent s'enfuir précipitamment, car la mêlée est susceptible 
de changer de position ou de direction à tout moment. Le public est d'ailleurs presque exclusivement masculin, si bien que la présence de femmes, non seulement parmi les joueurs, mais même parmi les spectateurs, est parfois mal vue.

Fig. 4. Spectateurs de tire-bouc, exclusivement des hommes, autour de la mêlée

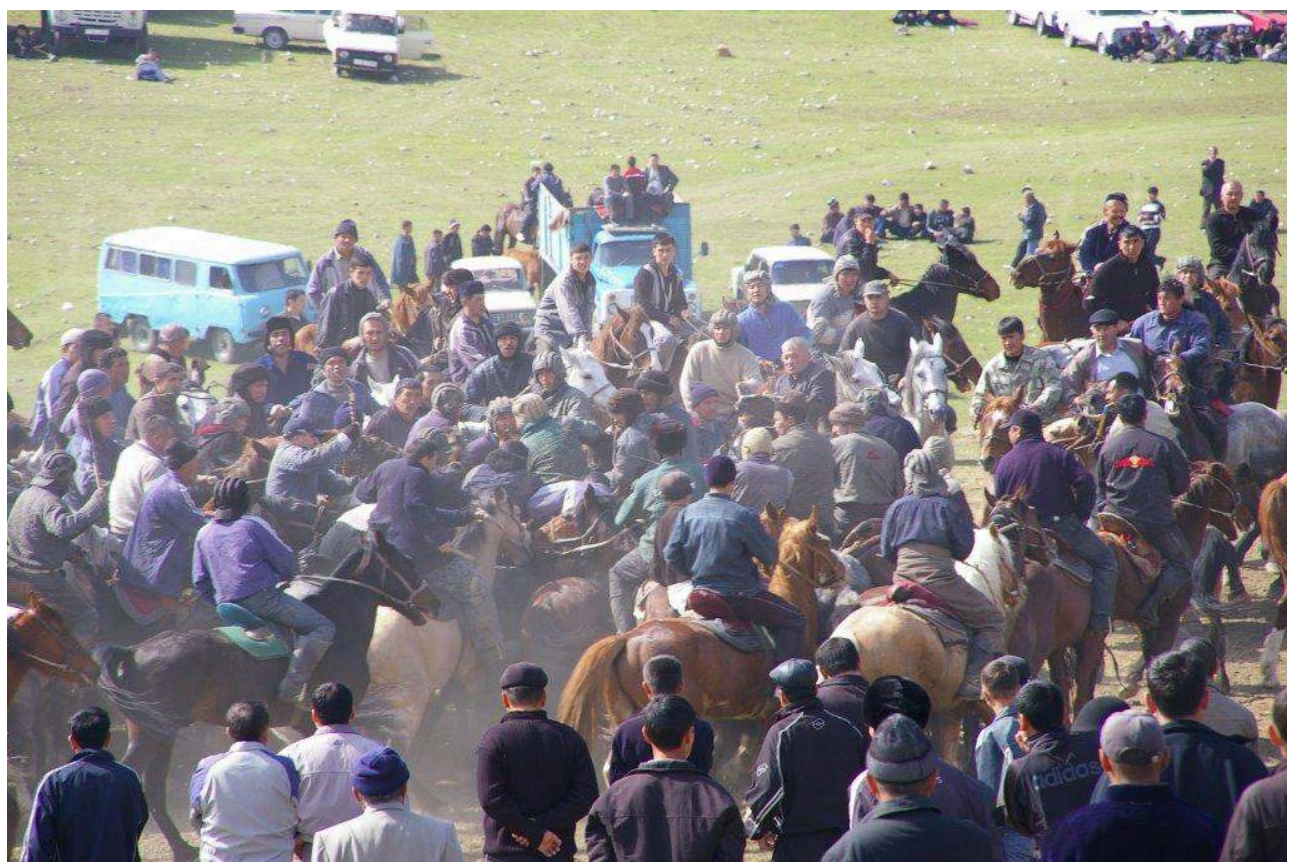

Photo C. Ferret, mars 2009, Ouzbékistan

La violence du tire-bouc constitue un premier obstacle qui s'oppose à sa mise en tourisme. Les mauvais traitements infligés aux animaux paraissent particulièrement malvenus dans un contexte où la sensibilité "animalitaire" ne cesse de croître en Occident depuis les années 1990, prônant un élevage respectueux de la bientraitance, dite "bien-être animal ", ou allant jusqu'à proscrire toute utilisation animale dans le mouvement vegan. Cette évolution a bouleversé le statut du cheval en Occident, le faisant passer de la sphère des animaux de rente, utilitaires, à celle des animaux de compagnie, et elle risque de condamner, à terme, toute pratique de l'équitation (Digard, 1995 ; 2004, p. 195 sq.).

Que dire alors d'une équitation où les chevaux sont fouettés, précipités les uns sur les autres et ont la bouche écorchée par le mors - sans parler de leur régime alimentaire qui, actuellement, n'indigne personne puisque les méthodes d'entraînement centrasiatiques sont totalement inconnues? Plusieurs controverses ont récemment agité les milieux des sports hippiques occidentaux sur des procédés condamnés comme relevant de la maltraitance, notamment le rollkur (hyperflexion de l'encolure) (McLean et McGreevy, 2010). L'usage de la cravache est réglementé (nombre de coups, position du bras, zones d'impact) et progressivement restreint dans les courses et les concours, désapprouvé par le public et des études scientifiques (Evans et McGreevy, 2011). En février 2019, un match de tire-bouc à l'hippodrome de Moscou a suscité la réprobation du public et le lancement d'une pétition par des défenseurs des animaux.

Quant aux usages du cheval en général, un malentendu révélateur d'un réel fossé culturel oppose, d'une part, ce que J.-P. Digard (2004) appelle des " sociétés à écuyers » occidentales, de plus en plus soucieuses du bien-être animal quitte à renoncer à 
l'équitation - qui n'est déjà plus qu'un loisir - et, d'autre part, des " peuples cavaliers ", où le cheval, polyvalent et omniprésent, conserve un rôle utilitaire, comme véhicule et fournisseur de produits, et n'est pas un objet de sensiblerie. L'ironie vient de ce qu'un cliché tenace prétend que les pasteurs centrasiatiques, qui vivraient en harmonie avec la nature, dans des communautés hybrides d'humains et de non-humains, considèrent leurs montures sur un pied d'égalité (Tiberghien et Xie, 2018, p.6). La déception des touristes occidentaux, séduits par cette image idyllique, qui est même parfois la motivation principale de leur voyage dans les steppes, est amère lorsqu'ils sont confrontés sur place au traitement quotidien des animaux. Il y a donc tout lieu de penser que ce fossé entrave l'intégration du tire-bouc parmi les sports hippiques internationaux, ainsi que son exploitation touristique.

\section{Un jeu indescriptible}

27 Un deuxième obstacle s'oppose à la mise en tourisme du tire-bouc: son manque de lisibilité. Lors des toj, un spectateur néophyte a du mal à comprendre ce qui se déroule devant lui tant le jeu est confus. Le simple fait de suivre des yeux le parcours du kôkpar n'est pas chose aisée, tant il est difficile à distinguer dans la mêlée. Au bout d'un moment, le bouc reste invisible, mais le spectateur devine qu'il se trouve là où la foule est la plus dense. Il ne voit pas non plus clairement où sont les buts, ni s'il y en a ; pas plus qu'il ne repère de jeu d'équipe. De temps à autre, c'est une clameur et la suspension momentanée du jeu qui lui donnent à penser qu'un essai a dû être marqué. Pire encore, dans la foule empoussiérée des cavaliers, il saisit mal qui est là pour jouer et qui est là pour regarder.

28 Cette illisibilité est due à plusieurs facteurs: l'absence de délimitation du terrain, l'entremêlement des participants au jeu et des spectateurs, la densité de la mêlée et l'extrême variabilité des règles du jeu. Le terrain, non circonscrit, est parfois accidenté. Les joueurs peuvent s'éloigner et sortir du champ de vision. Pour suivre le déroulement du jeu, il faut se placer en position surélevée : à cheval, juché sur un camion ou sur la pente d'une colline. Il n'y a aucune séparation entre les joueurs et le public, mais les spectateurs qui s'approchent du kôkpar sont à cheval, seule façon de s'y avancer sans risque. 
Fig. 5. Mêlée empoussiérée, sans séparation entre joueurs et spectateurs

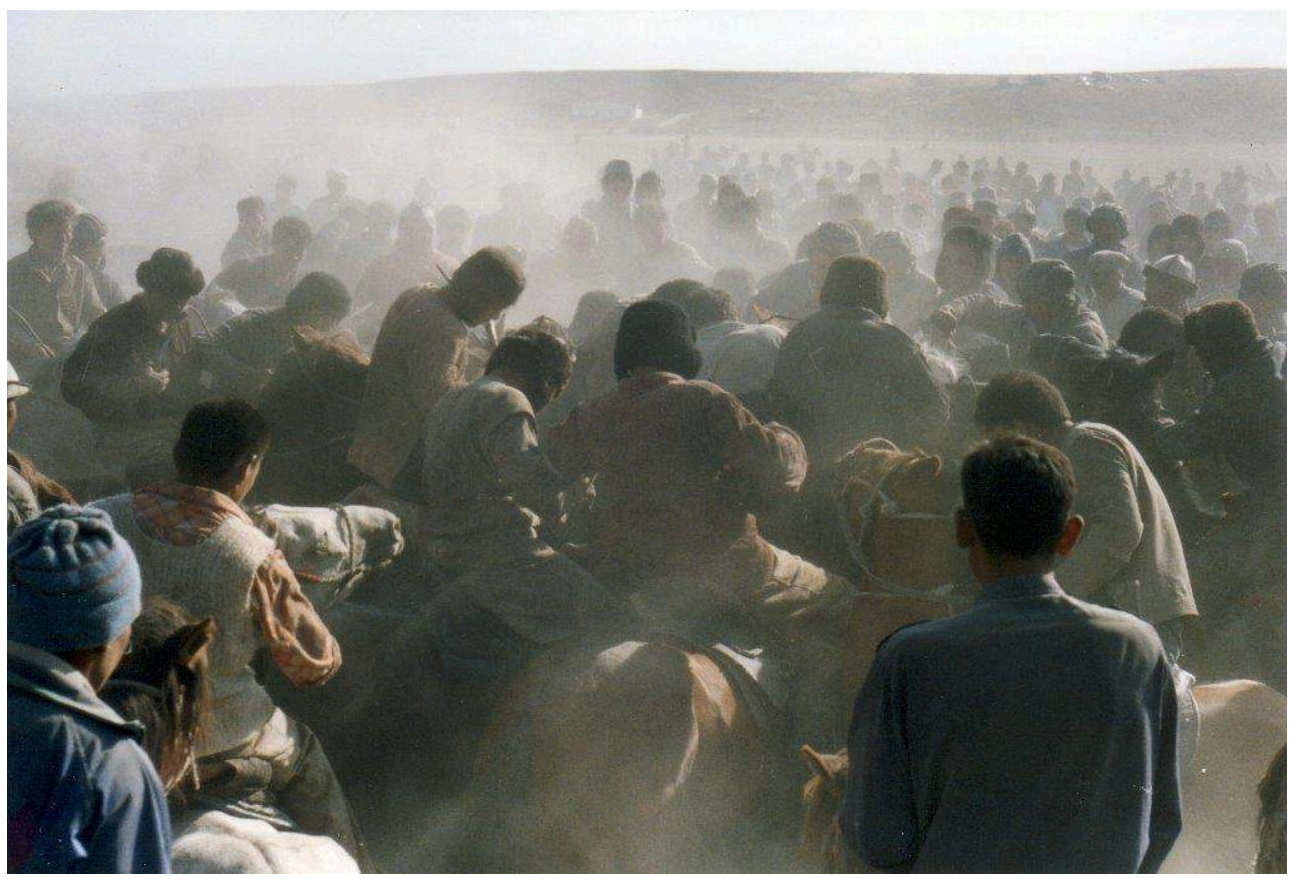

Photo C. Ferret, octobre 1994, sud du Kazakhstan

Le jeu manque aussi de visibilité en raison de la mêlée très serrée, qui forme une masse compacte, mi-humaine, mi-équine, où, comme au rugby, on ne voit plus guère quelle tête, quel bras ou quelle jambe appartient à quel corps, tel un amas de centaures démultipliés et agglutinés.

Fig. 6. Mêlée

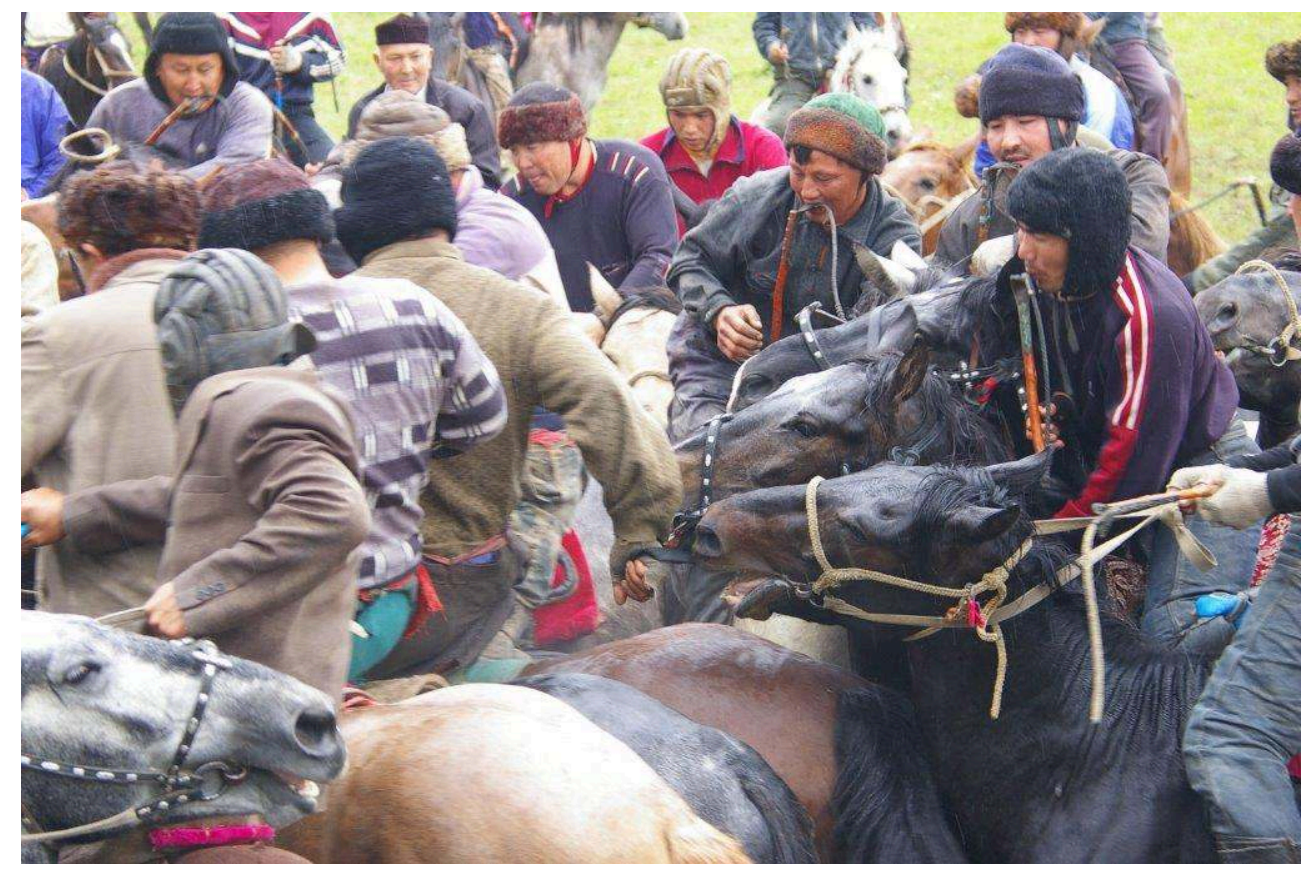

Photo C. Ferret, mai 2008, sud du Kazakhstan 


\section{tournoie ou se déplace par translation, chacun conservant au galop la même place par} rapport aux autres. Si un ŝabandoz parvient à s'extirper, le kôkpar coincé sous la cuisse, et distance ses adversaires, il peut gagner rapidement. Mais une autre fois, il sera rejoint, la mêlée se reformera autour de lui et il lui faudra de nouveau fournir le même effort pour l'emporter. Pas plus que le terrain, le temps de jeu n'est délimité. On joue sans s'arrêter jusqu'à marquer. Chaque assaut est donc de durée inégale, de quelques minutes à une demi-heure environ. Les buts ne sont pas comptabilisés, car chaque essai marqué donne droit à un prix, que le ŝabandoz et le propriétaire du cheval se partagent.

more de joueurs varie de quelques-uns à plusieurs centaines, jouant tantôt chacun pour soi (kaz. žalpy tartys), tantôt en équipe (doda tartys) - voire, dans la même partie, les uns jouant solo et les autres collectif. Pour couronner le tout, les équipes, quand elles existent, ne sont pas toujours au nombre de deux, ni ne comptent nécessairement le même nombre de membres, opposant, par exemple, un village à un autre. Enfin, le critère déterminant la victoire varie également. Quelquefois il suffit au šabandoz de prendre le large avec le kôkpar, mais le plus souvent, il doit le déposer en un endroit précis, un but marqué d'un cercle sur le sol. Les configurations sont, là encore, diverses : un seul but, le même pour tous; un but par équipe; ou encore deux buts entre lesquels les participants font des allers-retours lors des assauts successifs, tous en même temps dans la même direction.

seulement les règles du jeu connaissent de grandes variations, mais leur observance est loin d'être stricte. Les gestes ou procédés interdits, rappelés en début de partie (attacher le kôkpar à sa selle, fouetter la tête d'un cheval, désarçonner un adversaire en soulevant sa jambe, etc.) sont rarement sanctionnés s'ils sont transgressés, ce qui donne lieu à de longues discussions.

Heurtant les sensibilités occidentales et échappant à l'entendement en raison de son caractère chaotique, le tire-bouc, tel qu'il est joué dans les grandes fêtes centrasiatiques, ne réunit donc apparemment pas les qualités requises pour une exploitation touristique, en dépit d'un indéniable pouvoir de fascination, lié à son exotisme mais aussi, précisément, à ces deux grands défauts.

\section{...Métamorphosé en sport}

Les deux principaux obstacles à la mise en tourisme du tire-bouc, qui tiennent donc à sa violence et son illisibilité, ont été atténués par un long processus de sportification visant à transformer ce jeu en sport. Cette évolution est commune à de nombreuses activités ludiques et sportives. Mais l'originalité du tire-bouc tient à ce que, en Asie centrale, on n'observe pas un remplacement du jeu par le sport, mais une coexistence de ces différentes formes.

\section{Un jeu rituel}

Le tire-bouc se joue habituellement dans les toj, fêtes dispendieuses avec banquets et divertissements qui marquent la vie sociale des peuples centrasiatiques (Werner, 1999; Turdalieva et Provis, 2017). Les toj sont organisés en de multiples occasions, lors des rites de passage qui scandent les étapes du cycle de vie individuel : noces avant tout 
(kaz. ùjlenu toj, noce virilocale), mais aussi naissance, circoncision (kaz. sùndet toj), anniversaire décennal, etc. Les fêtes funéraires, également très nombreuses et constituant un cycle pour chaque défunt, forment un ensemble à part, où l'événement principal est l'as, célébré un an après le décès. Autrefois, les tire-bouc avaient lieu lors des toj et les bäjge lors des as, mais cette distinction s'est brouillée depuis la fin du XIX ${ }^{\mathrm{e}}$ siècle.

36 Toutes les familles n'ont pas les moyens d'organiser un tire-bouc pour célébrer un événement familial, car cela occasionne de grosses dépenses afin d'offrir des prix prestigieux aux vainqueurs (argent, bétail, véhicules, électroménager). Seuls les plus riches peuvent se le permettre, même si la plupart sont prêts à se ruiner pour monter une noce fastueuse, qui représente une obligation sociale impérieuse et un moyen essentiel d'asseoir sa notabilité. Les prix gagnés au tire-bouc sont partiellement redistribués et s'inscrivent dans un circuit de dons et de contredons, où donner est plus prestigieux que recevoir.

La spécificité de ces compétitions tient à ce que, d'une part, elles accompagnent des rites de passage et, d'autre part, l'honneur va à l'organisateur bien plus qu'aux vainqueurs. L'organisateur du toj, qui peut être le père du jeune marié ou du garçon circoncis, doit faire preuve de sa générosité par des dépenses ostentatoires : convier de nombreux invités, venus de toutes parts, à un banquet fastueux et offrir des prix de valeur aux vainqueurs du tire-bouc. C'est son nom qui sera proclamé et répété - car un bon toj se doit d'être mémorable -, accompagné de la liste des prix proposés, bien plus encore que celui des ŝabandoz victorieux (Ferret, 2018). Le tire-bouc, dans ces circonstances, peut être interprété comme un jeu rituel qui s'inscrit dans une économie festive constitutive des sociétés centrasiatiques (Light, 2015, p. 123 sq.).

En tant que jeu rituel, il combine des aspects du rite et de la performance. À propos du mariage tadjik, J. Cleuziou (2018) souligne la distinction entre rituel, qui implique une transformation de la personne, et performance, qui suppose une démonstration devant autrui. Elle entend la performance uniquement dans son sens théâtral, mais sa dimension sportive n'est pas à négliger. C'est précisément cet aspect-là du tire-bouc qui se prête le mieux à sa mise en tourisme. Transformer ce jeu rituel en spectacle sportif représentatif du patrimoine pastoral a donc été l'option choisie pour le valoriser, notamment auprès des touristes.

\section{Sa sportification}

La sportification du tire-bouc est antérieure aux indépendances des républiques d'Asie centrale. Elle s'inscrit dans un mouvement global de transformation des activités ludiques et violentes en sports dans l'Occident moderne (Elias et Dunning, 1994). En URSS, dès les années 1950, les règles du tire-bouc furent formalisées afin de le policer, comme furent aseptisés et folklorisés de nombreux jeux populaires des diverses nationalités. La variante du jeu en équipe était favorisée sur la lutte chacun pour soi (Gunner et Rahimgulov, 1949, p. 58). La normalisation des règles les rendait plus homogènes et moins versatiles. Des juges étaient chargés de surveiller leur observance. Le temps et l'espace de jeu étaient délimités. Les compétitions devaient avoir lieu sur des hippodromes, à défaut sur des terrains plats et circonscrits, dont les limites séparaient les joueurs, à l'intérieur, et le public, à l'extérieur. Le déroulement du jeu était soumis à un contrôle médical et vétérinaire qui devait en réduire la dangerosité. 
Des femmes étaient encouragées à y participer, ce qui n'a plus cours aujourd'hui (Bobylev, 1989, p. 29).

40 Ce mouvement de sportification du kôkpar s'est amplifié après les indépendances de 1991, avec la multiplication des compétitions, la refonte des règles et la création de fédérations au niveau national. Depuis, les règlements sont en constante évolution et varient selon les pays. Globalement, les tendances établies à l'époque soviétique ont été confirmées et accentuées : délimitation spatio-temporelle avec diminution de la taille du terrain, jeu par équipes avec réduction du nombre de joueurs en action. Comme auparavant, ces règles sont appliquées lors des compétitions sportives, mais pas dans les tire-bouc des toj, qui continuent à se jouer comme décrit plus haut et dont la popularité ne se dément pas.

41 Aujourd'hui, le tire-bouc sportif oppose deux équipes de 10 ou 12 joueurs, qui se relaient de telle sorte que seuls quatre sont engagés en même temps. Un match se joue en deux ou trois périodes de 20 minutes sur un terrain de 200x70 mètres aux extrémités duquel sont placés les buts de chaque équipe. L'équipe victorieuse est celle qui marque le plus de buts dans le temps réglementaire ${ }^{3}$. L'engagement se fait à mi-distance des deux buts, devant les tribunes, puis, à défaut, si personne n'a pu saisir le kôkpar dans les deux premières minutes, dans un cercle central où ne peuvent pénétrer que deux joueurs, un de chaque équipe, qui se livrent à ce que j'appelle un "duel de prise». Après une sortie de terrain en touche, l'engagement se fait également par un « duel de prise ». En cas de faute dans la surface de réparation située à proximité de chacun des deux buts, ou en cas d'égalité à l'écoulement du temps réglementaire (ou additionnel), on procède à quatre «duels au but » successifs (bullit), c'est-à-dire une poursuite entre deux joueurs distants de 30 mètres au départ, celui qui tient le kôkpar devant marquer sans être rejoint.

Cette nouvelle réglementation témoigne, par bien des aspects, d'une spectacularisation du jeu et de sa globalisation, en le rapprochant des sports collectifs parmi les plus populaires au monde, comme le football ou le hockey. Cette tendance se voit dans les maillots assortis de couleurs vives des équipes sportives, qui contrastent avec les tenues disparates aux tons neutres des ŝabandoz des toj, mais elle se décèle également dans les caractéristiques formelles du jeu. Ainsi la fermeture et la disposition du terrain, la confrontation égalitaire de deux équipes symétriques défendant chacune un but bien visible, précédé d'une "surface de réparation", la création de touches, de duels au but, équivalents des pénaltys et des tirs au but, sont calquées sur les sports de ballon.

43 L'innovation des duels opposant clairement deux couples cheval-cavalier accroit la visibilité du jeu par rapport au chaos de la mêlée. En outre, elle marque une tendance à l'individualisation et au vedettariat qui, par le biais des classements par points, braque les projecteurs sur quelques champions, humains et équins. 
Fig. 7. Championnat de la république d'Altaï

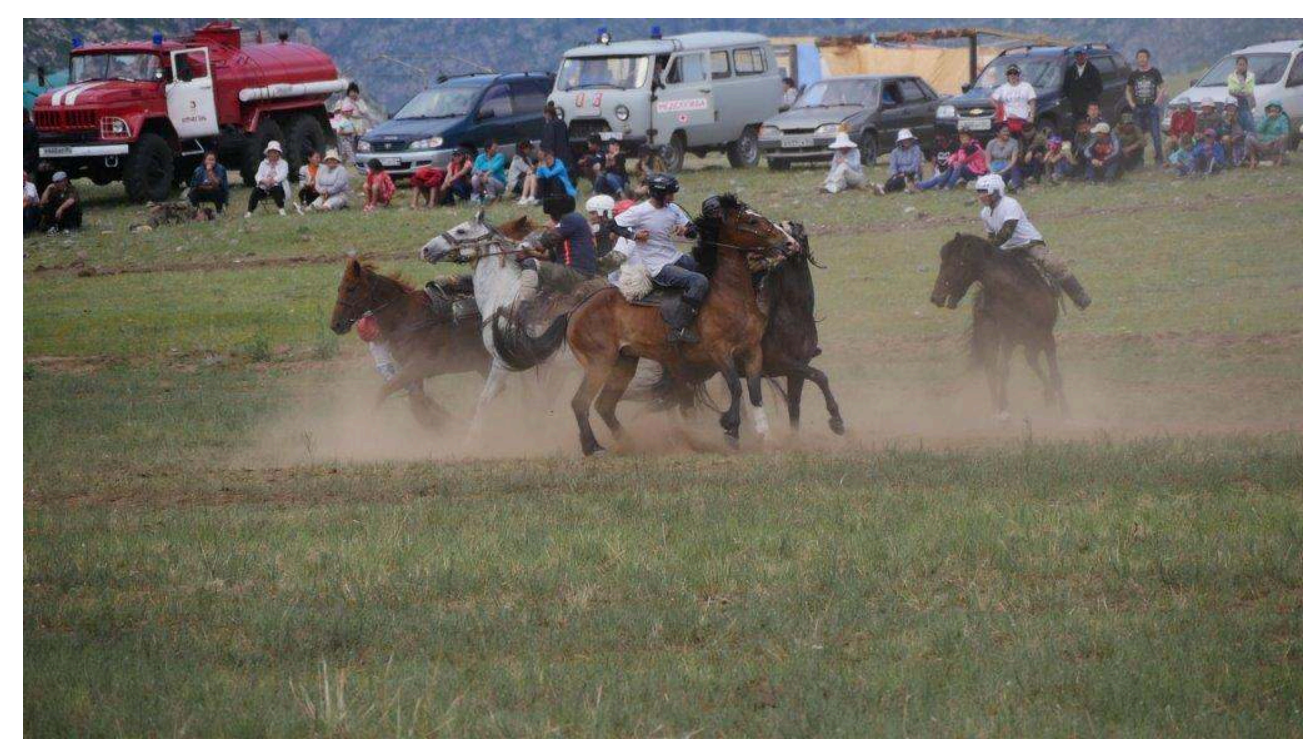

Le public, ici composé d'hommes et de femmes, est à distance des joueurs

Photo C. Ferret, juin 2017, Altaï, Russie

Plus d'un siècle après les sports anglais, le tire-bouc a donc subi une métamorphose similaire, à partir d'une lutte générale violente et chaotique vers un sport globalisé. A. Guttman (1978) cite sept critères qui ont concouru à l'émergence des sports modernes dans l'Angleterre du XVIII siècle. Ils s'avèrent tous à l'œuvre dans la sportification du tire-bouc: la sécularisation, par l'effacement de la signification rituelle; la rationalisation, par la formalisation de règles; la bureaucratisation, par l'organisation hiérarchique des fédérations sportives à divers échelons; la quantification, par l'enregistrement des scores; l'égalité, par l'instauration d'équipes ayant le même nombre de participants ; la spécialisation, par la division des rôles au sein d'équipes stables, entre défenseurs et attaquants, les uns se distinguant par leur force, les autres par leur rapidité ; enfin le record, par la création de championnats et le classement des équipes et des joueurs. De même, l'euphémisation de la violence que relèvent Elias et Dunning (1994) dans l'histoire de la lutte, du football ou de la chasse au

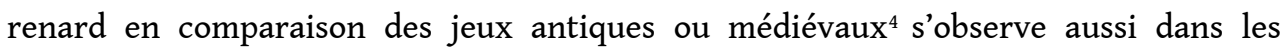
transformations du tire-bouc.

Mais l'analogie ne s'arrête pas là, dans les tendances générales de l'évolution du jeu. Les détails techniques de son déroulement montrent aussi à quel point les changements introduits correspondent parfois point par point aux transformations qu'ont connues d'autres sports auparavant, en particulier dans la délimitation spatio-temporelle du cadre de jeu (Bromberger, 1995). Outre une normalisation et une domestication de la violence, cette métamorphose se traduit par une mise en spectacle (Saumade, 2011).

P. Parlebas relève une rupture culturelle entre les jeux enfantins de la Renaissance, représentés par Bruegel en 1560 puis Stella en 1657, et les sports occidentaux contemporains. Or il est frappant de constater à quel point leurs différences formelles sont identiques à celles qui séparent le tire-bouc des toj de celui des compétitions sportives. Dans les premiers, les spectateurs sont absents ou mêlés aux joueurs, alors que l'institution sportive est "devenue une véritable entreprise de spectacles " où " acteurs et spectateurs sont radicalement séparés » (2003a, p. 17). Outre l'interdiction 
des comportements jugés violents, la codification, la quantification, la prise en compte du critère temporel, la création d'espaces dédiés et clos sont toutes des innovations du système sportif. Dans sa structure relationnelle, le tire-bouc a, comme les jeux de la Renaissance, évolué depuis le modèle d'un «jeu à réseau original» (un contre tous, chacun pour soi) vers un duel symétrique, caractéristique de l'affrontement sportif, entre deux équipes stables (ou parfois entre deux individus, lors des duels). Les équipes des tire-bouc des toj, même quand elles existent, ne sont ni stables ni symétriques. Simplement, dans le cas du tire-bouc, nous n'assistons pas aujourd'hui au remplacement d'un modèle par un autre, mais à la coexistence parallèle d'un jeu rituel et d'un sport spectacle, le second n'ayant pas évincé le premier.

\section{Objet de rivalité entre le Kirghizstan et le Kazakhstan}

Depuis quelques années, une rivalité croissante oppose le Kirghizstan et le Kazakhstan au sujet du tire-bouc. Le Kirghizstan a marqué des points décisifs, en organisant les "Jeux nomades mondiaux» à partir de 2014, puis en parvenant à faire inscrire le kôkbôrù au patrimoine immatériel de l'humanité par l'Unesco en 2017.

Les Jeux nomades sont à appréhender comme un "événement sportif fabriqué ${ }^{5}$ ", qui vise à construire l'image du pays par un savant dosage de sport et de patrimoine culturel et qui, ce faisant, favorise le développement touristique. Par son envergure, il se veut à la fois à niveau d'effet sociétal et à niveau d'échelle mondial, selon la typologie de Bessy et Suchet; il conviendrait néanmoins d'évaluer son rayonnement réel, qui ne dépasse vraisemblablement pas l'échelle aréale, malgré la présence de délégations venant de plusieurs continents.

49 La création des Jeux nomades a été précédée, à partir de 2005, de l'organisation, à l'initiative de la journaliste française Jacqueline Ripart, du festival At čabyš, promouvant le cheval kirghize par des courses d'endurance (Ferret, 2011a, p. 433-4). Les Jeux nomades ont une visée plus large, parce qu'ils regroupent plusieurs types de compétitions (course, lutte montée, tir à l'arc, chasse au vol, montage de iourtes, etc., le clou de la fête étant le kôkbôrù), mais aussi parce qu'ils revendiquent, pour le Kirghizstan, l'appropriation de l'héritage nomade à l'échelle mondiale.

50 L'octroi du label Unesco est une victoire à la fois plus restreinte, car elle se cantonne à ce jeu précis, et plus large, car elle est sanctionnée par un organisme international faisant mondialement autorité dans le domaine culturel. En assignant à une ethnie particulière le jeu du tire-bouc pratiqué dans une plus vaste aire culturelle, l'Unesco valide l'argumentaire du pays candidat et sert un enjeu nationaliste ${ }^{6}$. Toutes proportions gardées, sa politique culturelle n'est donc pas si éloignée de la politique soviétique des nationalités, qui créa des nations en Asie centrale en la divisant en cinq unités discrètes, les Républiques, différenciées entre elles et unifiées en leur sein (Ferret, 2011b, p. 460-1). Du point de vue kirghize, la labellisation Unesco permet d'espérer un effet positif sur le développement du tourisme, en combinant, comme pour les Jeux nomades, une dimension sportive et une dimension culturelle.

51 Sur le terrain du tire-bouc, le Kirghizstan et le Kazakhstan se livrent une bataille d'autant plus acharnée que Kirghizes et Kazakhs partagent, de fait, une même culture. Les premiers ont créé une "Fédération internationale de kôkbôrù ", mais les seconds ont répliqué en fondant de leur côté une "Association mondiale du kôkpar ». Les derniers championnats montrent que c'est souvent l'équipe du pays organisateur qui 
gagne, d'autant qu'elle joue chez soi suivant ses propres règles. Les divergences portent sur la ferrure des chevaux, la durée de jeu, la nature du kôkpar et la forme des buts. Plusieurs fois, l'équipe, kirghize ou kazakhe, du pays invité s'est fait exclure ou s'est retirée de la compétition, accusée d'être mauvaise perdante, car elle entendait suivre les règles qu'elle estime légitimes, à savoir les siennes.

Fig. 8. Lutte près d'un qazan

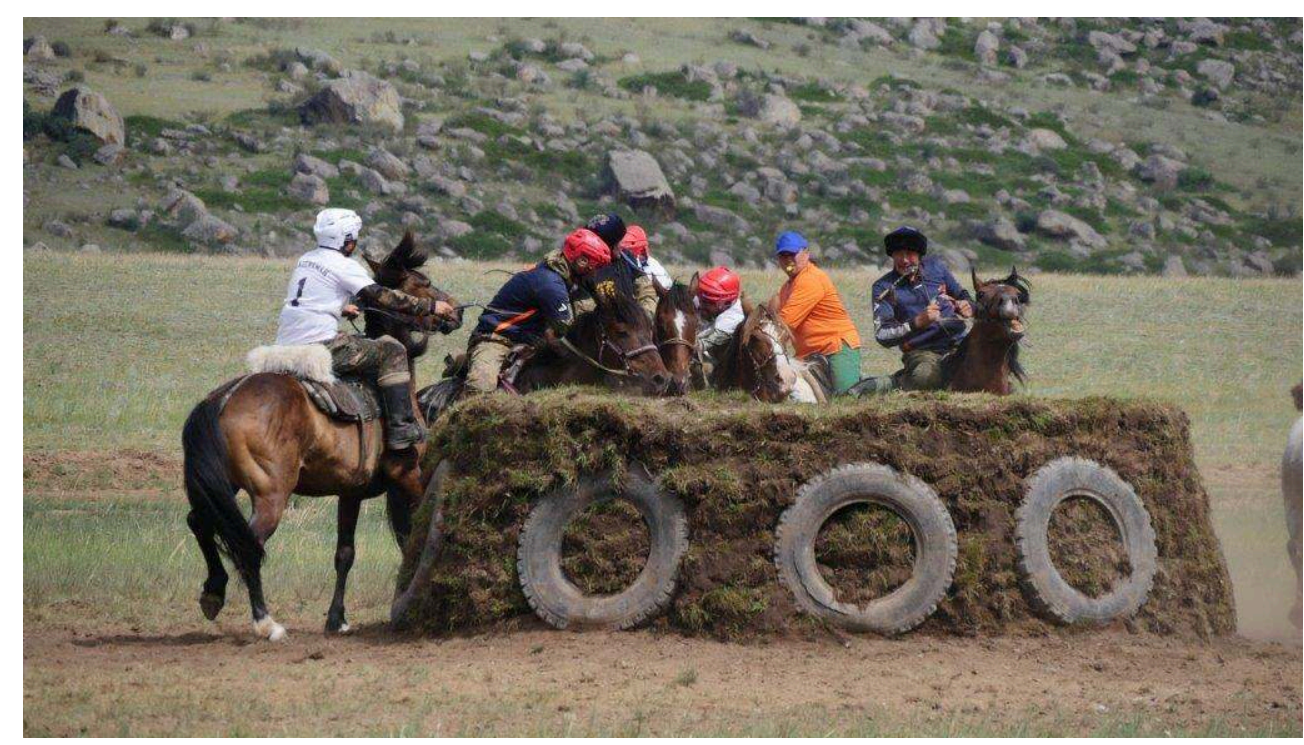

Photo C. Ferret, juin 2017, République de l'Altaï, Russie

Les points de discorde se concentrent sur la nature du kôkpar et surtout sur la forme des buts. Les Kazakhs prônent l'emploi d'un bouc factice en plastique pour ne pas heurter les défenseurs des animaux. Après les avoir utilisés quelques années, ils ont proscrit l'emploi des qazan, buts surélevés qui occasionnent de graves blessures. Ces tertres creusés en leur centre (d'une hauteur d'1,2 mètre et de 4 mètres de diamètre) transforment la nature du jeu, car ils nécessitent un effort supplémentaire pour marquer : les šabandoz doivent y lancer le kôkpar, et non simplement le laisser tomber dans un cercle marqué au sol. Pour les Kirghizes, qui les ont introduits, les qazan présentent l'avantage de rendre le jeu plus spectaculaire. En 2016, ils les ont remplacés par des armatures plus légères, afin de réduire leur dangerosité. En 2017, la fédération kazakhstanaise a néanmoins décidé de les interdire. Les journalistes vont jusqu'à parler d'un « schisme " (rus. raskol) à ce propos car, en février 2019, les pays participants ont décidé de suivre les règles kazakhes du kôkpar et non les règles kirghizes du kôkbôrù pour le prochain championnat du monde qui devait avoir lieu à Tachkent en novembre 2019. À la suite de cette décision, le Kirghizstan a annoncé qu'il refuserait de participer. Et à l'automne, le championnat a été reporté.

Ces litiges n'aident pas à l'internationalisation du jeu. Mais ce conflit entre Kirghizes et Kazakhs est révélateur des tensions qui se manifestent à l'occasion de choix techniques. Pour promouvoir ce jeu, en faire un sport internationalement reconnu et attirer les touristes, faut-il en atténuer la brutalité ou en accentuer le côté spectaculaire? Ce débat kirghizo-kazakh sur les règles du jeu renvoie aux deux inconvénients majeurs qui font du tire-bouc un jeu bien peu touristique : sa brutalité et son manque de visibilité. 
Cette dispute fournit aussi un bel exemple d'invention des traditions, thème classique en sciences sociales depuis Hobsbawm et Ranger (1983). Nous avons déjà évoqué l'imposture qui consiste à présenter le tire-bouc comme un jeu "nomade ». Mais dans les querelles sur les détails techniques se manifeste aussi une bataille d'authenticité. Les Kirghizes reprochent aux Kazakhs de trahir le jeu en renonçant à la tradition des qazan, oubliant de préciser que ces derniers sont une invention récente, datant de 1996.

Le réalisateur de cinéma Bolot Šamšiev, mort en décembre 2019, a participé à plusieurs étapes d'un vaste plan de promotion de la culture kirghize par le biais de grands événements équestres et patrimoniaux. En 1994, il planifiait un raid équestre dit "Super marathon », puis les " Mille miles de la Route de la soie ». En 1995, il a œuvré à la fastueuse célébration des 1000 ans de l'épopée Manas (Thompson et al., 2006). En 1996, il a participé à la rédaction des nouvelles règles du kôkbôrù. Son projet de « Djiguit show ", ou «Cow-boy show ", à l'image des rodéos américains", s'est concrétisé sous la forme des Jeux nomades quelques années plus tard.

Comme le montre le rôle de Šamŝiev, le tire-bouc présente un cas exemplaire d'une entreprise de développement du tourisme culturel et de marchandisation de la culture, qui passe par sa transformation en spectacle exotique pour un monde globalisé. Ainsi, le réalisateur explique clairement les principes qui ont présidé aux nouvelles règles du kôkbôrù :

Auparavant, il n'y avait pas de buts et le terrain n'était pas clos, les cavaliers pouvaient partir loin, à 5 kilomètres. Ils jouaient pour eux-mêmes. Mais c'est pour le spectateur qu'il faut jouer. Seul un réalisateur de cinéma pouvait inventer ce jeu au format nouveau, quelqu'un habitué au contact avec les spectateurs et qui connaît la psychologie du spectateur. ${ }^{8}$

Cette spectacularisation assumée est néanmoins une arme à double tranchant : à force de scénarisation, les démonstrations de tire-bouc pourraient apparaitre comme une prestation folklorique, artificiellement montée à l'unique intention des touristes, qui n'entendent pas se laisser ainsi berner (Tiberghien et Xie, 2018, p. 7-8).

\section{Conclusion}

58 La sportification du tire-bouc est-elle un bon choix dans une stratégie de développement touristique? Faire de ce jeu brutal et chaotique un spectacle sportif régulé est une option qui paraît cohérente, pour atténuer les deux défauts qui risquent d'entraver son exploitation touristique: sa violence, susceptible de heurter les sensibilités, et son manque de lisibilité, qui le rend incompréhensible.

9 Cependant, ce choix appelle plusieurs remarques. Premièrement, dans une optique de spectacularisation du jeu, le touriste est un simple spectateur ${ }^{9}$, alors qu'il est acteur car pratiquant la randonnée sportive - dans la stratégie de l'écotourisme, jusqu'à présent dominante au Kirghizstan et, dans une moindre mesure, au Kazakhstan. Cette passivité du touriste spectateur aurait d'ailleurs été la même si l'accent avait été mis sur la dimension rituelle du tire-bouc, plutôt que sur sa dimension sportive.

Deuxièmement, les deux traits évoqués, violence et étrangeté, s'ils apparaissent comme des inconvénients, sont aussi des facteurs de la fascination qu'exerce ce jeu. La violence fait partie de l'attrait du tire-bouc (Guttman, 1998). «Le sport [...] est une forme de combat qui donne du plaisir sans choquer la conscience " (Elias, 1976, p. 21), à condition de trouver le bon dosage, en fonction des seuils de tolérance de chacun. Mais 
il n'est pas certain qu'un juste équilibre puisse être atteint, entre sensibilité animalitaire et désir d'authenticité. En outre, la perte de signification rituelle et sociale de ce jeu métamorphosé en sport émergent et sa standardisation à l'aune des jeux de ballon mondialisés ne nuisent-elles pas à son inscription dans un cadre patrimonial ?

Troisièmement, les aléas de la sportification et la controverse kazakho-kirghize sur les règles du jeu révèlent de profonds tiraillements entre tradition et modernité. Elles montrent l'ambivalence d'un héritage nomade, brandi souvent à tort et pourtant pas totalement assumé. Paraphrasant Werner (2000), cet article aurait pu s'intituler "Consuming modernity, imagining tradition: Globalization, nationalism and... sport ».

\section{BIBLIOGRAPHIE}

G. Whitney AzoY, Bozkashi : jeu et pouvoir en Afghanistan, Le Capucin, 2002.

Harold B. BARCLAY, The role of the horse in man's culture, J.A. Allen \& Co, 1980.

Olivier BESSY et André SUCHET, « Une approche théorique de l'événementiel sportif », Mondes du tourisme, $\mathrm{n}^{\circ}$ 11, 2016 [https://journals.openedition.org/tourisme/1023].

I.F. BOBYLEV, Konnye igry [Les jeux équestres], Mehnat,1989.

Christian BROMBERGER, « De quoi parlent les sports? » Terrain, n² 25, 1995.

Alexander BURNES, Cabool: a personal narrative of a journey to and residence in that city, J. Murray, 1842.

Juliette CLEUZIOU, « De faux amis ? Les concepts de rituel et de performance à l'épreuve d'une ethnographie du mariage au Tadjikistan ", Thaêtre, ch. 3, 2018.

Jean-Pierre DIGARD, Une histoire du cheval : art, techniques, société, Actes Sud, 2004.

Jean-Pierre DIGARD, «Cheval, mon amour. Sports équestres et sensibilités “animalitaires” en France ", Terrain, n 25, 1995.

Louis DUPREE, Afghanistan, Princeton University Press, 1980 [1973].

Norbert ELIAS, «Sport et violence ", Actes de la recherche en sciences sociales, vol. 2, n 6, 1976.

Norbert ELIAS et Eric DUNNING, Sport et civilisation : la violence maîtrisée, Fayard, 1994 [1986].

David EVANS et Paul MCGREEVY, « An Investigation of Racing Performance and Whip Use by Jockeys in Thoroughbred Races », Plos One, vol. 6, n 1, 2011.

P.A. FEDOTOV, Nacionnal'nye konnosportivnye igry [Les jeux équestres sportifs des nationalités], Kajnar, 1980.

Carole FERRET, « Le kôkpar, un jeu sérieux. Démêlage d'une mêlée hippique centrasiatique », ethnographiques.org, $n^{\circ}$ 36, 2018 [https://www.ethnographiques.org/2018/Ferret].

Carole FERRET, « Les ambiguïés du patrimoine nomade des Kazakhs », Nomadic Peoples, vol. 20, $n^{\circ} 2,2016$. 
Carole FERRET, « À chacun son cheval! Identités nationales et races équines en ex-URSS (à partir des exemples turkmène, kirghize et iakoute) », Cahiers d'Asie centrale, $n^{\circ} 19-20,2011 \mathrm{a}$.

Carole FERRET, « L'identité, une question de définition », Cahiers d'Asie centrale, n 19-20, 2011b.

Carole FERRET, « Course à la mort ou quête de respectabilité : le bäjge en Asie centrale », Ethnozootechnie, $n^{\circ}$ 82, 2008.

Carole FERRET, « De l'attache des chevaux à la fécondation des femmes en passant par la cuisine. Quelques pistes pour l'exploration des notions altaïques de chaud et de froid », Études rurales, $\mathrm{n}^{\circ} 171,2004$.

M. GUNNER et M. RAHIMGULOV, Kratkij sbornik kazahskih narodno-nacional'nyh vidov sporta [Précis des sports nationaux et populaires kazakhs], Kazahskoe ob. gos. izd.,1949.

Allen GUTTMAN, « The appeal of violent sports », dans J. GOLDSTEIN (dir.), Why We Watch: The Attractions of Violent Entertainment, Oxford University Press, 1998.

Allen GUTTMAN, From ritual to record: the nature of modern sports, Columbia University Press,1978.

Julie HALLÉ et Michel RASPAUD, « Les guides de montagne d'Asie centrale et l'activité de tourisme sportif. Effets et conséquences de la transition socio-économique ", Mondes du tourisme, $\mathrm{n}^{\circ}$ 6, 2012 [https://journals.openedition.org/tourisme/245].

E.J. HOBSBAWM et T.O. RANGER, The Invention of tradition, Cambridge University Press, 1983.

Rico ISAACS, « Nomads, warriors and bureaucrats: nation-building and film in post-Soviet Kazakhstan », Nationalities Papers, vol. 43, n 3, 2015.

Joseph KESSEL, Les cavaliers, Gallimard, 1967.

V.P. KolosovsKIJ, Lošadi Turkestana [Les chevaux du Turkestan], Izd. Turkestanskogo ob. pooŝreniâ konnozavodstva, 1910.

Elizabeth A. LAWRENCE, Rodeo, an anthropologist looks at the wild and the tame, University of Tennessee Press, 1982.

Nathan LIGHT, « Self-Sufficiency is Not Enough: Ritual Intensification and Household Economies in a Kyrgyz Village », dans S.F. GUDEMAN et C. HANN (dir.), Oikos and market: explorations in selfsufficiency after socialism, Berghahn, 2015.

Andrew N. MCLEAN et Paul D. MCGREEVY, « Horse-training techniques that may defy the principles of learning theory and compromise welfare », Journal of Veterinary Behavior: Clinical Applications and Research, vol. 5, $\mathrm{n}^{\circ}$ 4, 2010.

Johanne PABION-MOURIÈS, «Tourisme communautaire et patrimonialisation de la culture. Le cas du Kirghizstan postsoviétique et multiethnique », Collection EDYTEM. Cahiers de géographie, $\mathrm{n}^{\circ} 14$, 2013.

Pierre PARLEBAS, « Une rupture culturelle : des jeux traditionnels au sport », Revue internationale de psychosociologie, vol. IX, $\mathrm{n}^{\circ} 20,2003 \mathrm{a}$.

Pierre PARLEBAS, « Le destin des jeux : héritage et filiation », Socio-anthropologie, $\mathrm{n}^{\circ} 13,2003 \mathrm{~b}$ [https://journals.openedition.org/socio-anthropologie/173.]

Frédéric SAUMADE, «Le rodéo américain sur la frontière du sport et de la tauromachie, ou de la diffusion considérée comme un système de transformations ", Ethnologie française, vol. 41, $\mathrm{n}^{\circ} 4$, 2011. 
G.N. SIMAKOV, Obŝestvennye funkcii kirgizskih narodnyh razvlečenij v konce XIX - načale XX v. [Les fonctions sociales des distractions populaires kirghizes (fin $\mathrm{XIX}^{\mathrm{e}}$ - début XX⿳⺈冂大 $\mathrm{s}$.)], Nauka, 1984.

K.J. THOMPSON, P. SCHOFIELD, N. FOSTER et G. BAKIEVA, « Kyrgyzstan's “Manas” Epos Millennium Celebrations: Post-Colonial Resurgence of Turkic Culture and the Strategic Marketing of Cultural Tourism ", dans D. PICARD et M. ROBINSON (dir.), Festivals, Tourism and Social Change: Remaking Worlds, Channel View Publications, 2006.

Guillaume TIBERGHIEN et Philip Feifan XIE, « The life cycle of authenticity: neo-nomadic tourism culture in Kazakhstan ", Journal of Tourism and Cultural Change, vol. 16, n 3, 2018.

Cholpon tURDALIEVA et Rene PROVIS, « Dynamics of Reciprocity and Networks of the Kyrgyz through Bishkek Toi Making ", Central Asian Affairs, vol. 4, n² 2, 2017.

Cynthia WERNER, « The new silk road: Mediators and tourism development in Central Asia », Ethnology, vol. 42, n², 2003.

Cynthia WERNER, « Consuming modernity, imagining tradition: Globalization, nationalism and wedding feasts in postcolonial Kazakstan », Anthropology of East Europe Review, vol. 18, n², 2000.

Cynthia WERNER, « Dynamics of feasting and gift exchange in rural Kazakstan », dans I. SVANBERG

(dir.), Contemporary Kazaks: cultural and social perspectives, St. Martin's Press, 1999.

\section{NOTES}

1. L'Asie centrale stricto sensu se compose de cinq républiques de l'ex-URSS: Kazakhstan, Kirghizstan, Ouzbékistan, Tadjikistan, Turkménistan, dont les ethnies titulaires et majoritaires sont turcophones, à l'exception du Tadjikistan, iranophone.

2. Voir : https://www.populationdata.net (consulté le 7 octobre 2019).

3. Pour le règlement au milieu des années 1990, voir Ferret, 2018. La victoire a successivement été accordée : 1) au premier ŝabandoz qui marquait (sans critère temporel); 2) à la première équipe qui marquait sept buts (score-limite) ; 3) à l'équipe qui marquait le plus dans un temps donné (« temps-limite ») (cf. Parlebas, 2003a, p. 20).

4. Le football, par exemple, était qualifié de « fureur bestiale » en 1531 (Elias, 1976, p. 16).

5. Bessy et Suchet (2016) définissent l'événement sportif par la conjonction de cinq éléments : organisation préalable, limite de temps et de lieu, médiatisation, performance sportive et retombées. Les événements fabriqués, nés localement, s'opposent aux événements importés, au format prédéfini. Ces auteurs distinguent le niveau d'effet, qui qualifie l'emprise de l'événement, et le niveau d'échelle, qui correspond à la dimension (locale, régionale ou mondiale) de son rayonnement.

6. L'Unesco n'attribue pas explicitement le kôkbôrù aux seuls Kirghizes, mais en associant ce jeu au nom du Kirghizstan et en choisissant cette dénomination-ci à l'exclusion des autres, l'effet est le même.

7. Communication personnelle en mai 1994; interview du 11 juin 2006 lue sur: http:// www.kyrgyznews.com/news.php?readmore $=1261$

8. Voir : http://topsport.kg/article/show/130/

9. J'ai trouvé un tour operator qui propose de participer à des kôkpar, mais c'est tout à fait exceptionnel, car ce jeu est affaire de spécialistes. 


\section{RÉSUMÉS}

Le jeu de tire-bouc (kôkpar en kazakh, kôkbôrù en kirghize), dont le bouzkachi afghan est une des variantes, est pratiqué par plusieurs peuples turciques d'Asie centrale. C'est une sorte de rugby à cheval, où le cadavre d'un caprin tient lieu de ballon. Au Kirghizstan et au Kazakhstan, il a pu être présenté comme un élément du patrimoine nomade susceptible d'attirer les touristes par son exotisme. Cette assertion est cependant le fruit de plusieurs malentendus, dus à la méconnaissance de ce jeu violent, dont il existe actuellement des formes diverses. Jeu rituel, organisé pour des fêtes marquant les étapes du cycle de vie, il participe d'une économie festive qui confère du prestige à l'organisateur plus qu'au vainqueur. Il existe parallèlement sous la forme d'une compétition sportive, issue d'un processus de sportification entamé au milieu du $\mathrm{xx}^{\mathrm{e}}$ siècle, qui se traduit par une formalisation et une transformation de ses règles, à propos desquelles se disputent les deux pays rivaux. De ces deux grandes formes de tire-bouc, jeu rituel et sport émergent, laquelle siéra le mieux à des touristes en quête d'authenticité, avides de spectacles et dotés d'une sensibilité plus « animalitaire » que celle de leurs hôtes?

Goat-pulling (kôkpar in Kazakh, kôkbôrù in Kyrgyz), otherwise named Afghan buzkashi, is a game practiced by several Turkic peoples in Central Asia. It is a kind of rugby on horseback played with a dead goat instead of a ball. In Kyrgyzstan and Kazakhstan, it is supposedly part of the nomadic heritage, whose exoticism could attract tourists. This allegation is nevertheless caused by several misinterpretations, due to unfamiliarity. This violent game presently exists in various forms. As a ritual game organized for life-cycle feasts, it takes part in the ritual and festive economy and gives prestige to the host organizer rather than to the winner. In parallel, it is also played as a sport competition, emerging from a sportification process which had consisted in the formalisation and the transformation of its rules since the middle of the $20^{\text {th }}$ century. Which of these two main forms of goat-pulling (ritual game or emerging sport) will better suit to tourists seeking authenticity, eager to see spectacular performances and at the same time more sensitive to animal welfare than their Central-Asian hosts?

\section{INDEX}

Mots-clés : Asie centrale, Kazakhstan, Kirghizstan, sportification, jeu équestre, cheval, identité nationale touristique, tire-bouc, bouzkachi

Keywords : Central Asia, Kazakhstan, Kyrgyzstan, sportification, equestrian game, horse, touristic national identity, goat-pulling, buzkashi

\section{AUTEURS}

\section{CAROLE FERRET}

Chargée de recherche au CNRS

Laboratoire d'anthropologie sociale (CNRS - Collège de France - EHESS)

carole.ferret[at]college-de-france.fr

\section{CAMILLE NOÛS}

Laboratoire Cogitamus

camille.nous[at]cogitamus.fr 